\title{
QUALIDADE MICROBIOLÓGICA DE ALIMENTOS SERVIDOS EM RESTAURANTES UNIVERSITÁRIOS
}

\author{
MICROBIOLOGICAL QUALITY OF FOOD SERVED IN UNIVERSITY RESTAURANTS
}

\author{
Miguel Augusto Lopes Neto ${ }^{1 *}$, João Victor de Sousa Osti ${ }^{1}$, Bruna da Silva Rocha ${ }^{1}$, Lidiane Pinto de \\ Mendonça ${ }^{2}$
}

${ }^{1}$ Acadêmicos do curso de Nutrição da Faculdade Nova Esperança de Mossoró (FACENE/RN) *miguellopes735@gmail.com
${ }^{2}$ Nutricionista, docente da Faculdade Nova Esperança de Mossoró (FACENE/RN)

\section{Info}

Recebido: 06/2020

Publicado: 09/2021

DOI: $10.37951 / 2358-260 X .2021 v 8 \mathrm{i} 2.5813$

ISSN: 2358-260X

Palavras-Chave

Alimentos; Restaurantes Universitários;

Microrganismos; Manipuladores.

Keywords:

Food; University Restaurants;

Microorganisms; Handlers.

\section{Resumo}

Para a portaria $\mathrm{N}^{\circ} 326$, de 30 julho 1997 o alimento quando realizado desde a matéria prima animal ou vegetal, estão sujeitos a sofrer contaminação, fermentação ou apodrecimento. $\mathrm{Na}$ sua maioria, os alimentos têm riquezas de nutrientes e elevada atividade de água, com isso, uma boa qualidade higiênico-sanitária do local e dos manipuladores deve mantê-los livres de contaminação. Dentro da microbiologia, existem diversos tipos de microrganismos, que podem propiciar melhores características organolépticas dos alimentos e podendo conviver com simbiose dentro do organismo humano. Nesse sentido, levando em consideração a periculosidade das doenças transmitidas por alimentos, como também o elevado consumo de alimentos fora de casa, objetivou-se verificar a qualidade microbiológica de alimentos servidos em restaurantes universitários através de uma revisão integrativa. As amostras foram encontradas a partir de pesquisas nas bases de dados PubMed, Scielo e Google Acadêmico em busca de artigos científicos captados de uma triagem de publicações segundo as palavras chaves: "restaurante universitário", "análise microbiológica", "cantinas", "alimentos", "manipulação de alimentos" e "condições higiênico sanitárias". Os estudos são referentes a análise microbiológica de alimentos em restaurantes universitários, de caráter experimental desenvolvidos. Nos estudos analisados foram encontrados contaminação por coliformes totais e termotolerantes, salmonella spp. e staphylococcus spp. indicando má manipulação. Por não existir uma fiscalização eficaz, pode-se constatar que os restaurantes universitários agem sem responsabilidade com os clientes quanto a manipulação do alimento. Portanto, faz necessário medidas que possam vir a treinar e capacitar a equipe dessas unidades de alimentação, para que assim, aconteça uma manipulação segura, livre de contaminantes e para que seja adequada para o consumo humano.

\footnotetext{
Abstract

According to Ordinance No. 326, of July 30,1997, when food is made from animal or vegetable raw material, it is subject to contamination, fermentation or rotting. Most of the foods are rich in nutrients and have high water activity, so a good hygienic-sanitary quality of the place and the handlers should keep them free from contamination. Within microbiology, there are several types of microorganisms, which can provide better organoleptic characteristics of food and can coexist with symbiosis within the human body. In this sense, taking into account the danger of foodborne diseases, as well as the high consumption of food outside the home, the objective was to verify the microbiological quality of food served in university restaurants through an integrative review. The samples were found from searches in the PubMed, Scielo and Google Academic databases in search of scientific articles collected from a selection of publications according to the key words: "university restaurant", "microbiological analysis", "canteens", "food ", "food handling" and "sanitary hygienic conditions". The studies refer to microbiological analysis of foods in university restaurants, with an experimental character developed. In the analyzed studies, contamination by total and thermotolerant coliforms, salmonella spp. and staphylococcus spp. indicating poor handling. Because there is no effective inspection, it can be seen that university restaurants act without responsibility with customers regarding food handling. Therefore, it is necessary to take measures that can train and qualify the staff of these food units, so that safe handling can take place, free of contaminants and so that it is suitable for human consumption.
} 


\section{INTRODUÇÃO}

A Declaração Universal dos Direitos Humanos reconhece a alimentação, em seu Artigo XXV, como um direito humano básico e a Constituição de 1988 reafirma que a alimentação é um direito de todos. O alimento propicia inúmeros papéis fundamentais para o bom funcionamento do organismo, através do fornecimento de vitaminas e minerais.

Para a portaria $\mathrm{N}^{\circ}$ 326, de 30 julho 1997 o alimento quando realizado desde a matéria prima animal ou vegetal, estão sujeitos a sofrer contaminação, fermentação ou apodrecimento. $\mathrm{Na}$ sua maioria, os alimentos têm riquezas de nutrientes e elevada atividade de água, com isso, uma boa qualidade higiênicosanitária do local e dos manipuladores deve mantê-los livres de contaminação.

Uma alimentação de qualidade, não se resume apenas a sua composição bioquímica, mas consiste também em isenção de microrganismos contaminantes. Um dos parâmetros fundamentais em ambientes alimentícios é a segurança alimentar, que trata da isenção do contato do alimento com qualquer tipo de ação microbiológica, interação esta que acaba ocasionando danos à saúde humana ao consumir este alimento (SILVA, 2014). Para isso, no Brasil foi instituída a Lei de Segurança Alimentar que dispõe sobre a realização do direito de todos ao acesso regular e permanente a alimentos de qualidade, em quantidade suficiente, sem comprometer o acesso a outras necessidades essenciais, tendo como base práticas alimentares promotoras de saúde, que respeitem a diversidade cultural e que sejam ambiental, cultural, econômica e socialmente sustentáveis.

Segundo o guia alimentar da população brasileira, a persistência da globalização no século XXI possibilita um acelerado ritmo de vida e a busca pela praticidade tornou-se habitual. Por esse motivo, as pessoas optam cada vez mais por alimentar-se fora de casa, em busca de maior funcionalidade. Levando em consideração a rotina de um estudante universitário, a sua busca pela praticidade está diretamente ligada aos RU's -Restaurantes Universitários, sendo esse, o local onde os estudantes fazem a maior parte de suas refeições diárias (BRASIL, 2014).

A Agência Nacional de Vigilância Sanitária (ANVISA), relata por meio da Resolução da Diretoria Colegiada - RDC no 216, de setembro de 2004, que os serviços de alimentação devem seguir procedimentos de boas práticas a fim de garantir as condições higiênico sanitárias, desde a avaliação criteriosa dos fornecedores, passando por cuidados durante o recebimento, armazenamento, higienização do ambiente, higiene pessoal, produção, exposição do alimento e o descarte.

De acordo com a Organização Pan-americana de Saúde (2015), a falta de segurança alimentar esta adjunto à morte de cerca de 2 milhões de pessoas por ano. Os alimentos podem conter microrganismos responsáveis por mais de 200 doenças, desde diarreia ao câncer gerando um problema de saúde pública. São vários os fatores dentro de um estabelecimento contribuintes para tais fatos, como por exemplo a má higienização do local, o controle inadequado por parte dos manipuladores, erros no processo de preparação e serviço das refeições.

Dentro da microbiologia, existem diversos tipos de microrganismos, que podem propiciar melhores características organolépticas aos alimentos e podendo conviver com simbiose dentro do organismo humano. Porém, é possível ainda, ser encontrados microrganismos com um alto grau de patogenicidade, que quando ingerido pelo homem podem causar inúmeras adoecimentos e até mesmo morte, mais conhecida como doenças transmitidas por alimentos (FRANCO; LANDGRAF, 2005).

Nesse sentido, levando em consideração a periculosidade das doenças transmitidas por alimentos, 
como também o elevado consumo de alimentos fora de casa, objetivou-se verificar a qualidade microbiológica de alimentos servidos em restaurantes universitários através de uma revisão integrativa.

\section{METODOLOGIA}

A pesquisa realizada trata-se de uma revisão integrativa de caráter quali-quantitativo, definido por Souza e colaboradores (2010), como um método que proporciona a síntese de conhecimento e a incorporação da aplicabilidade de resultados de estudos significativos na prática.

A pesquisa foi realizada de agosto a dezembro de 2020. As amostras foram encontradas a partir de pesquisas nas plataformas digitais PubMed, Scielo e Google Acadêmico em busca de artigos científicos captados de uma triagem de publicações segundo as palavras chaves: "restaurante universitário", "análise microbiológica", "cantinas", "alimentos", "manipulação de alimentos" e "condições higiênico sanitárias". No rastreamento das publicações foi utilizado o operador lógico “AND”, de modo a combinar os termos acima citados.

Para a triagem de artigos foram considerados os seguintes critérios de inclusão: artigos publicados em português e inglês, disponíveis na íntegra nas bases de dados selecionadas, no período de 2012-2020, cuja a metodologia adotada fossem estudos descritivos experimentais. Foram excluídos estudos de revisões.

A seleção dos artigos se deu em três etapas: $1^{\circ}$ Etapa: leitura dos títulos; $2^{\circ}$ Etapa: leitura dos resumos; $3^{\circ}$ Etapa: leitura na íntegra.

\section{RESULTADOS E DISCUSSÃO}

$\mathrm{Na}$ busca 534 artigos foram encontrados, e após a leitura dos títulos foram excluídos 519 artigos resultando em 15 trabalhos. Após a leitura dos resumos foram extraídos apenas 6 estudos. A terceira fase dispõe sobre a leitura integral dos artigos restantes, no qual apenas 4 seguiram para a elaboração da revisão, por atender a todos os critérios de inclusão. Esses dados se encontram no fluxograma 1.

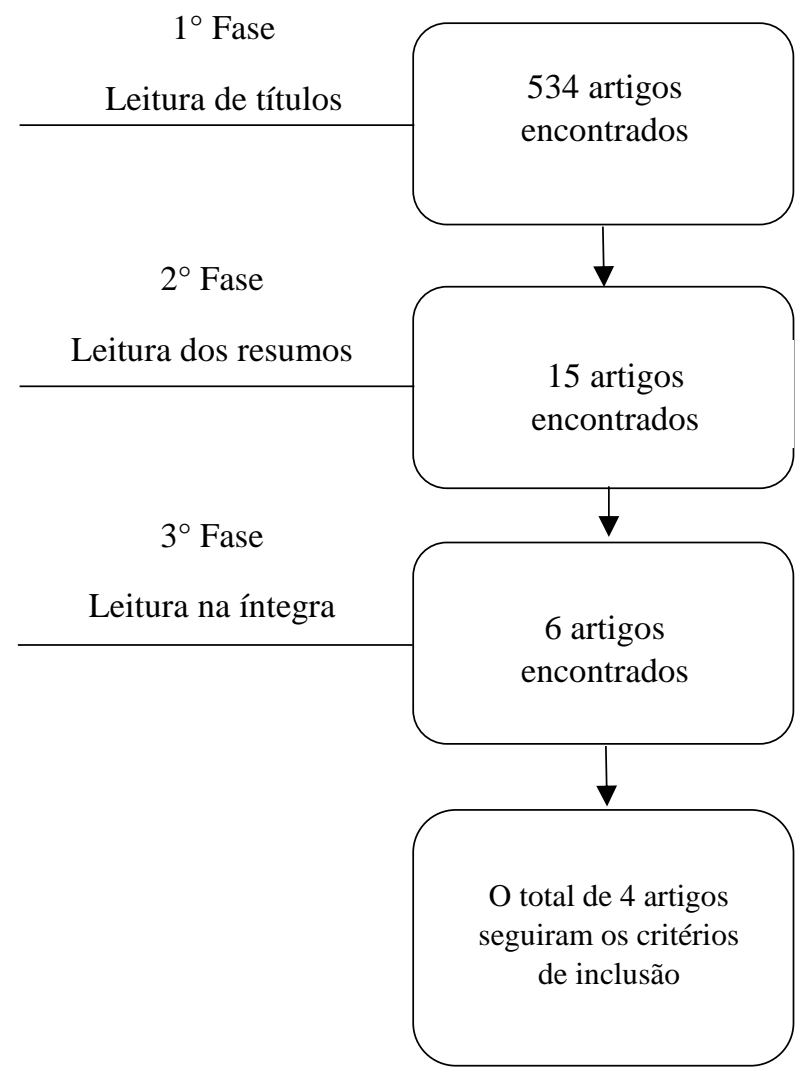

Figura 1: processo de seleção dos artigos publicados sobre a análise microbiológica de microrganismos em restaurantes universitário.

Para a revisão integrativa foram analisados 4 estudos que atenderam os critérios de inclusão previamente estabelecidos. Os estudos são referentes a análise microbiológica de alimentos em restaurantes universitários. Foram incluídos 1 estudo de 2012, 1 de 2015, 1 de 2017 e 1 de 2019. Esses dados se encontram na tabela 1. 
Tabela 1: Trabalhos selecionados referentes a análise microbiológica de alimentos em restaurantes universitários

TÍTULO

REFERÊNCIA
MICRORGANISMOS

ENCONTRADOS
Qualidade microbiológica

da água de consumo

humano e dos alimentos

comercializados em

lanchonete universitária
Coliformes a $45^{\circ} \mathrm{C}$

Fungos F. e Leveduras

Estafilococos Coagulase Positiva

Salmonella spp.

\section{Análise microbiológica de saladas e água servidas em um restaurante universitário do Triângulo Mineiro, Minas Gerais, Brasil}

$\begin{array}{ll}\text { Lima et al, 2015 } & \text { Coliformes totais } \\ \text { Coliformes termotolerantes }\end{array}$

\section{Avaliação das condições \\ microbiológicas de alimentos, superfícies e utensílios utilizados no preparo de refeições em um restaurante universitário}

Faria et al, 2012

\section{Análise microbiológica de sanduíches naturais comercializados na cidade de Fortaleza-CE}

Rodrigues et al, $2017 \quad \begin{aligned} & \text { Coliformes totais } \\ & \text { Coliformes termotolerantes } \\ & \text { Staphylococcus }\end{aligned}$

Os agravos à saúde resultantes da ingestão de alimentos impróprios ao consumo humano representam, atualmente, um dos mais disseminados e crescentes problemas de Saúde Pública. Doenças Transmitidas por Alimentos são definidas como aquelas usualmente de natureza infecciosa ou tóxica causada por agentes que invadem o organismo através da ingestão de alimentos (SILVA, 2009).

Lima e colaboradores (2015), analisaram 10 amostras de saladas prontas servidas em um restaurante universitário, utilizando a técnica de número mais provável (NMP). Os resultados das saladas analisadas mostraram contaminação variando entre $<3$ a $>1100$ NMP/g para coliformes termotolerantes, no qual, 40\% das destas estavam acima do que preconiza a RDC $\mathrm{n}^{\circ}$ 12 de janeiro de 2001 (até $100 \mathrm{NMP} / \mathrm{g}$ ). A salada que apresentou maiores níveis de contaminação por coliformes termotolerantes foi a de almeirão, alface e uva passa $(>1100 \mathrm{NMP} / \mathrm{g})$.

Além disso, os autores realizaram a análise de coliformes totais, no qual foram encontrados em todas as amostras de saladas valores iguais ou superiores a $1000 \mathrm{NMP} / \mathrm{g}$. Mesmo não havendo limites estabelecidos na legislação vigente, esses dados servem como indicadores de contaminação fecal, além de condições higiênico-sanitárias insatisfatórias.

Os coliformes totais e termotolerantes são microrganismos indicadores, utilizados para fornecer informações de contaminação de origem fecal, incluindo a presença de patógenos e a deterioração do alimento, além de indicar as condições higiênicosanitárias inadequadas. São compostos por bactérias da 
família Enterobacteriaceae, que por 48 horas são capazes de fermentar lactose com produção de gás, quando incubados a $35-37^{\circ} \mathrm{C}$. Já os coliformes termotolerantes, são comumente denominados de coliformes fecais, sendo estes pertencentes a um subgrupo de coliformes totais (FRANCO, LANDGRAF, 2005). Também possuem capacidade de continuar fermentando lactose com produção de gás, mas em temperaturas mais elevadas de $44-45,5^{\circ} \mathrm{C}$.

A tabela 2 mostra informações acerca dos microrganismos encontrados no trabalho analisado.

Tabela 2: Análise microbiológica de saladas cruas em restaurantes universitários

\begin{tabular}{ccc}
\hline Microrganismo & $\boldsymbol{N}^{\circ}$ de amostras & $\%$ encontrado \\
\hline Coliformes totais & $4 / 10$ & $40 \%$ \\
Coliformes termotolerantes & $4 / 10$ & $40 \%$ \\
\hline
\end{tabular}

Faria e colaboradores (2012), analisaram em seu estudo 8 amostras de alimentos, sendo eles 'Esfiha de carne', 'Coxinha de frango', 'Pão de queijo' e 'Suco de laranja' colhidos de acordo com maior frequência de saída e foram analisados em 2 momentos distintos. Para a análise de coliformes a $45^{\circ} \mathrm{C}$, a primeira amostra apresentou valores menores que 3,0 NMP/g, resultado esse que foi repetido na segunda testagem. Estes valores, segundo a RDC n⿳12/2001, indica níveis satisfatórios e livres de contaminação, mostrando assim, que houve bons métodos higiênicos-sanitários por meio dos manipuladores.

Frota e colaboradores (2019), analisaram 20 amostras de sanduíches naturais em pontos comerciais de uma universidade. Para coliformes a $45^{\circ} \mathrm{C}$ foram encontrados valores insignificantes variando entre $<3$ a $43 \mathrm{NMP} / g$. Porém para coliformes a $35^{\circ} \mathrm{C}$, mesmo não havendo parâmetro estabelecido pela RDC n¹2/2001, houve contaminação de $100 \%$ das amostras, indicando falhas no processo de fabricação.

Rodrigues e colaboradores (2017), avaliaram 32 tipos de preparações de alimentos em um restaurante universitário, escolhidos aleatoriamente, no qual quinzenalmente eram colhidas amostras que eram oferecidas no almoço e no jantar, constando porções frias e quentes, logo após 1 hora em que cubas foram abertas para o consumo. Foram analisados saladas cruas e cozidas e carnes, dentre essas, as saladas cruas e cozidas foram as que mais apresentaram níveis de coliformes totais, acima da recomendação proposta pela RDC n¹2/2001. Para coliformes termotolerantes os níveis encontrados são baixos e seguros para o consumo humano, segundo as recomendações da RDC no12/2001.

Os Staphylococcus spp. são da família Microccaceae, anaeróbicos facultativos, cocos grampositivos, imóveis, não produzem esporos e catalase positiva de alta distribuição. São muito comuns e responsáveis por surtos de intoxicação alimentar, por serem facilmente transmitidos através de alimentos. Ao ingerir o alimento contaminado, ocorre a intoxicação, advinda das enterotoxinas pré-formadas, produzidas pela bactéria, gerando sintomas diversos, que podem ser confundidos com intoxicações alimentares de outros microrganismos. Esses contaminantes podem contaminar pessoas saudáveis ou não, além de objetos inanimados, via contato direto ou através de gotículas (PRADO et al., 2015).

Faria e colaboradores (2012), em sua pesquisa, não encontrou a presença de Staphylococcus (ECP) na 
primeira etapa, no qual estudou esfiha de carne, coxinha de frango, pão de queijo e suco. Já na segunda fase para uma maior comprovação, foram analisados os mesmos alimentos e constatou-se que a coxinha de frango indicou a contagem de $1,0 \times 10^{3} \mathrm{UFC} / \mathrm{g}$ e na esfiha de carne, 2,0x103 UFC/g, havendo contaminação a níveis tóxicos para o homem, pois segundo a RDC n¹2/2001 o limite máximo permitido para Estafilococos coagulase positiva é de $10^{3} \mathrm{UFC} / \mathrm{g}$.

A tabela 3 mostra informações acerca dos microrganismos encontrados no trabalho analisado.

Tabela 3: Análise de Staphylococcus coagulase positiva da segunda etapa de quantificação.

\begin{tabular}{cccc}
\hline Alimentos & Quantidade & Recomendado pela RDC & Resultado \\
& & $\mathbf{n}^{\mathbf{0} 12 / 2001}$ & \\
\hline Coxinha de frango & $1,0 \times 10^{3} \mathrm{UFC} / \mathrm{g}$ & $10^{3} \mathrm{UFC} / \mathrm{g}$ & Presente \\
Esfiha de carne & $2,0 \times 10^{3} \mathrm{UFC} / \mathrm{g}$ & $10^{3} \mathrm{UFC} / \mathrm{g}$ & presente \\
\hline
\end{tabular}

Fonte: ADAPTADO de Farias et al. (2012).

Para Frota e colaboradores (2019), todas as amostras analisadas apresentaram valores inferiores a 10,0 UFC/g, ou seja, satisfatórios para Staphylococcus coagulase positiva, indicando um alimento seguro e com boas condições higiênico-sanitárias. Já para Rodrigues e colaboradores (2017), os níveis nas saladas cruas e cozidas e a carne de boi com mandioca foram significativos, quando comparados com os propostos pela RDC n¹2/2001. Mostrando assim, que houve níveis insatisfatórios no processo de manipulação higiênica.

No estudo de Faria e colaboradores (2012), foi percebido a presença de contaminação por Salmonella spp, mostrando assim, que os produtos selecionados para análise estavam inseguros para serem comercializados segundo a RDC n ${ }^{\circ} 12 / 2001$. Porém de maneira análoga, Frota e colaboradores (2019), identificou a ausência de contaminação por essa mesma bactéria.

A salmonella spp. é uma bactéria gram-negativa anaeróbia facultativa e não produtora de esporos, neste grupo existe vários sorotipos encontrados que podem vir acometer a saúde humana. As bactérias salmonella typhi e a paratyphi A e B são as mais graves e facilmente encontradas em surtos, pois o principal reservatório é o trato gastrointestinal dos homens e animais, sendo mais comum em aves (PRADO et al., 2015). Em todo o mundo, o consumo da proteína animal é muito comum, fazendo-se presente nas grandes refeições de toda família. A carne animal, quando má manipulada e/ou preparada, pode ser o principal veículo de contaminação por Salmonelose.

O Bacillus cereus bactéria produtora de endósporos, responsável pela produção de enterotoxinas que provocam diferentes sintomas no corpo humano, são elas na forma emética, na qual os sintomas são praticamente náuseas e vômitos e na forma diarreica, causando diarreia e dor gastrointestinal. É comum em alimentos cozidos e mantido em temperatura ambiente para o resfriamento como o arroz, massas, carnes ou molhos. Essas toxinas só são produzidas quando os endósporos germinam (MADGAN et al, 2016).

Para Frota e colaboradores (2019), em suas análises todas as amostras coletadas apresentavam-se satisfatórias, apresentando valores abaixo de $5 \times 10^{3}$ $\mathrm{UFC} / \mathrm{g}$, quando comparado com os limites estabelecidos pela RDC n ${ }^{\circ} 12 / 2001$. 
A RDC 12/2001 não impõe limite de contaminação de origem fúngica para os alimentos salgados, apenas para doces em pasta, purês, geléias e doces em calda, que apresenta tolerância de até $1,0 \times 10^{4}$ UFC/g. Nesse sentido, Faria e colaboradores (2012), sobre a análise de fungos filamentosos e leveduras, observou em seu estudo a quantificação destes microorganismos no pão de queijo (1,65 x 104 UFC/g) e no suco de laranja $\left(1,1 \times 10^{4} \mathrm{UFC} / \mathrm{g}\right)$, no qual apresentouse um pouco elevada. Já na esfiha de carne e na coxinha de frango, as contagens se mostram baixas aos níveis indicados como tóxico pela RDC n¹2/2001.

Pertencentes ao reino fungi, os fungos apresentam alta plasticidade genética e versatilidade fisiológica, são capazes de produzir muitos esporos que podem se espalhar por grandes extensões e são capazes de permanecer inertes por anos. São um grupo bem eficiente quanto a adaptação e sobrevivência, que não dependem da luz e por isso também podem ocupar locais escuros. Crescem em qualquer direção e são capazes de invadir o interior dos substratos com hifas responsáveis pela absorção. Com relação a sua morfologia, existem dois tipos que são: leveduras e hifas. (FRANCO, LANDGRAF, 2005).

No gráfico 1 consta os microrganismos encontrados nos estudos analisados.

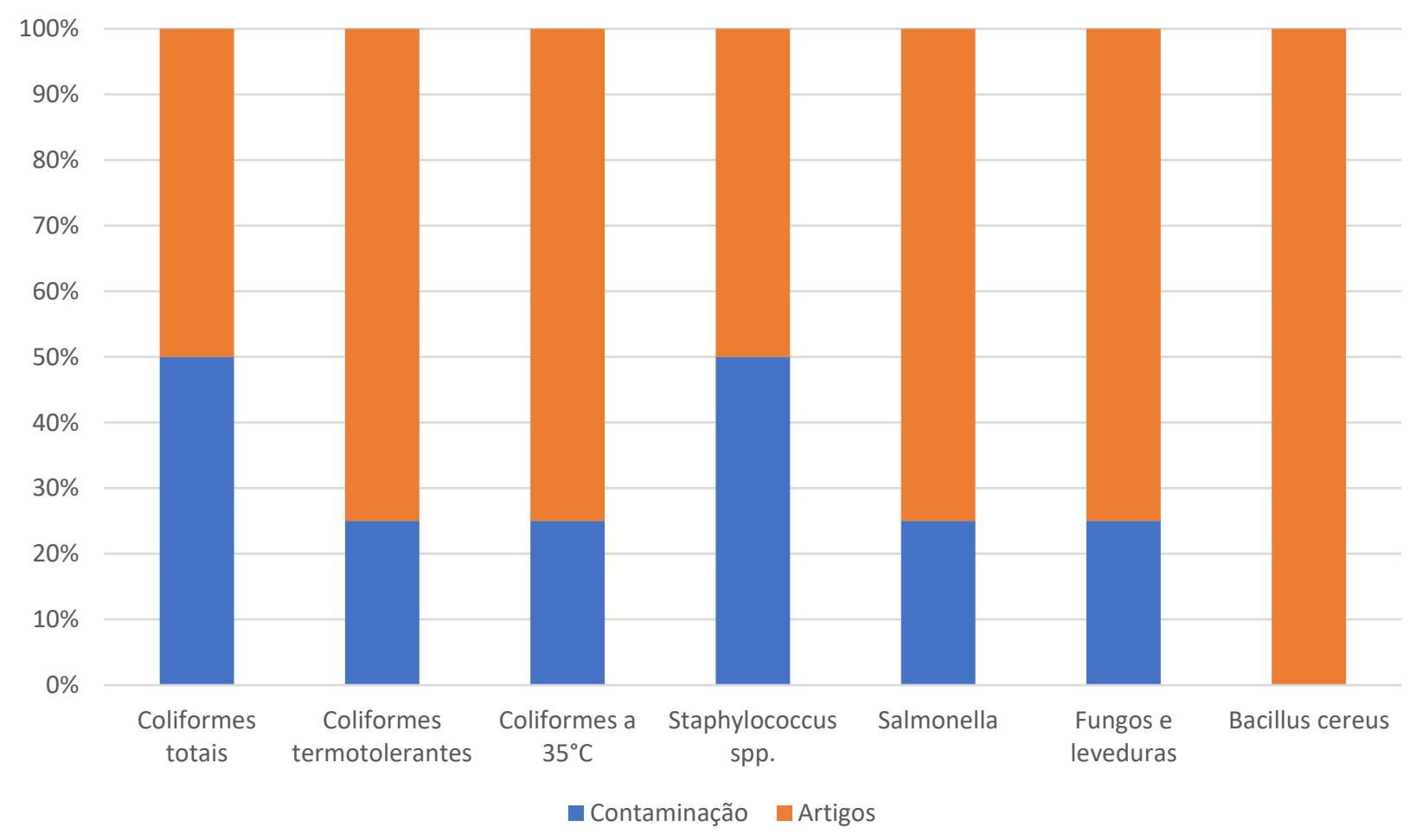

Figura 1: Porcentagem de contaminação referente aos microrganismos.

\section{CONSIDERAÇÕES FINAIS}

Os resultados encontrados nessa pesquisa mostram uma má manipulação de alimentos e consequentemente uma contaminação por parte de microrganismos como coliformes totais e termotolerantes, salmonella spp. e staphylococcus spp. Por não existir uma fiscalização eficaz, pode-se constatar que os restaurantes universitários agem sem responsabilidade com os clientes quanto a manipulação do alimento, mostrando assim, que ainda se faz necessário medidas que possam vir a treinar e capacitar a equipe dessas unidades de alimentação, para que assim, aconteça uma 
manipulação segura, livre de contaminantes e para que seja adequada para o consumo humano.

Portanto, levando em consideração o exposto, essa pesquisa enfatiza a importância e necessidade de controle microbiológico de alimentos em restaurantes universitários.

\section{REFERÊNCIAS}

BRASIL, Ministério da saúde, Agência Nacional de Vigilância Sanitária (ANVISA), Resolução RDC n 12 de 02 de janeiro de 2001. Regulamento Técnico Sobre Os Padrões Microbiológicos para Alimentos. Diário Oficial. Brasília, DF. 10 de janeiro de 2011.

BRASIL. Ministério da Saúde. Guia alimentar para a população brasileira / Ministério da Saúde, Secretaria de Atenção à Saúde, Departamento de Atenção Básica. - 2. ed. 156 p. Brasília: Ministério da Saúde, 2014.

COZZOLINO, S. M. F. Biodisponibilidade de Nutrientes. 5 ed. São Paulo: Manole, 2016.

FROTA, M. L. G., RIBEIRO, J. B., DOS SANTOS, S. F., OLIVEIRA, E. S, REIS, R. P. C., DA COSTA, M. T. P. ANÁLISE MICROBIOLÓGICA DE SANDUÍCHES NATURAIS COMERCIALIZADOS NA CIDADE DE FORTALEZA-CE, 2019.

FARIA, T., DE OLIVEIRA PAULA, R. A., DE LIMA GERMANO, J., OLIVER, J. C., DE ÂLCANTARA, B. G. V., VIEIRA, C. R., \& VEIGA, S. M. O. M. Qualidade microbiológica da água de consumo humano e dos alimentos comercializados em lanchonete universitária. Revista da Universidade Vale do Rio Verde, 10(2), 360369, 2013.

LANDGRAF, M. Microrganismos Indicadores. In: FRANCO, B. D. G. M; LANDGRAF, M. Microbiologia dos alimentos. São Paulo: Editora Atheneu, 2005.

LIMA, E. D. N. S., MENDES, R. A., DO AMARAL, A. B.,DE FÁTIMA CARRIJO, K. Análise microbiológica de saladas e agua servidas em um restaurante universitário do Triangulo Mineiro, Minas Gerais, Brasil. Rev.
Enciclopédia Biosfera, 11(22), 3176-3185, 2015.

MADIGAN, M. T. et al. Microbiologia de Brock. 14. ed. Porto Alegre: Artmed, 2016.

Organização Mundial da Saúde. (1997, 30 de junho). Regulamento técnico sobre as condições higiênico-sanitárias e de praticas de fabricação para estabelecimentos produtores/ industrializadores de alimentos. Disponível em: http://bvsms.saude.gov.br/bvs/saudelegis/svs 1/1997/prt0326_30_07_1997.html. Acesso em: 15 set. 2020.

PRADO, R.Resende et al. Staphylococcus spp.: importantes riscos à saúde pública. PUBVET, v. 9, p. 348-399, 2015.

RODRIGUES, J. M., DARDAQUE, R. M., FURLANETO-MAIA, L., GOMES, J. P., DE OLIVEIRA, A. F. Avaliação das condições microbiológicas de alimentos, superfícies e utensílios utilizados no preparo de refeições em um restaurante universitário, 2017.

SILVA, J. Manual de Controle Higiênico-Sanitário em Serviços de Alimentação. 7ed. São Paulo: Livraria Varela; 2014.

SILVA, Y. et al. Doenças transmitidas por alimentos no município do Rio de Janeiro: perfil epidemiológico e controle. 2009. Tese de Doutorado.

SOUZA, M. T.; SILVA, M. D.; CARVALHO, R. Revisão integrativa: o que é e como fazer. Einstein (São Paulo), v. 8, n. 1, p. 102-106, 2010. 